\title{
A New Method to Treat Severe Asymptomatic Pre- existing Degeneration of Adjacent Segment: a Retrospective Case-control Study
}

Wenjie Gao (D 419494560@qq.com )

xi'an honghui hospital

\section{Xinliang Zhang}

Xi'an Honghui Hospital

Jinwen Zhu

Xi'an Honghui Hospital

Zhen Chang

Xi'an Honghui Hospital

Yibing Li

Xi'an Honghui Hospital

Xiaodong Wang

Xi'an Honghui Hospital

Dingjun Hao

Xi'an Honghui Hospital

Research article

Keywords:

Posted Date: July 30th, 2019

DOl: https://doi.org/10.21203/rs.2.12097/v1

License: (c) (i) This work is licensed under a Creative Commons Attribution 4.0 International License. Read Full License 


\section{Abstract}

Background Pre-existing degeneration of adjacent segment is an important risk factor for adjacent segment degeneration (ASD), but limited and controversial studies have addressed its management.

Methods Patients with symptomatic degeneration of the L5/S1 segment warranting surgical interference and severe asymptomatic degeneration of the $L 4 / 5$ segment were retrospectively analyzed. Among them, those who underwent interbody fusion in the causative (L5/S1) segment and distraction of the intervertebral space and facet fusion in the adjacent $L 4 / 5$ segment were included as Group $A(n=103)$. Patients who underwent interbody fusion in both $L 5 / S 1$ and $L 4 / 5$ segments were included as Group B $(n=81)$. Clinical and radiographic outcomes were evaluated. Results Mean follow-up was 58.5 months (range, 48-75 m). No significant difference in clinical outcomes or the incidence of adjacent segment degeneration in L3/4 segment was found between Groups A and B. Compared with Group B, less bleeding ( $315 \pm 84$ vs. $532 \pm 105 \mathrm{ml})$, shorter operation time (107 \pm 34 vs. $158 \pm 55 \mathrm{~min}$ ) and lower costs $(13,830 \pm 2640$ vs. $16,020 \pm 3380$ US\$) were found in Group A ( $<<0.05)$. In Group A, disc height ratio (DHR) of $L 4 / 5$ segment was significantly increased from preoperative value of $0.40 \pm 0.13$ to last follow-up value of $0.53 \pm 0.18(P<0.05)$, while the degree of canal stenosis (DCS) was decreased from preoperative value of $34.3 \pm 11.2 \%$ to last follow-up value of $15.9 \pm 9.3 \%(P<0.05)$. Conclusions This new method could be effective in treating severe asymptomatic pre-existing degeneration of adjacent segment in lumbar spine.

\section{Background}

Posterior lumbar interbody fusion (PLIF), which can provide posterior fusion concomitant with anterior column support, is an effective procedure for lumbar degenerative diseases such as lumbar disc herniation, degenerative spondylolisthesis, degenerative instability and spinal canal stenosis. However, with its wide applications, many complications emerge, including pseudarthrosis, loosening or breaking of the implant, and adjacent segment degeneration (ASD). Development of ASD has attracted increasing attention because it adversely affects long-term clinical outcome and sometimes requires surgery[1-3]. The exact cause of ASD remains controversial, yet some risk factors have been documented[4,5]. Among them, pre-existing degeneration of the adjacent segment is one of the most important risk factors $[1,4,6]$. Studies concerning whether asymptomatic pre-existing degeneration of adjacent disc should be treated, and how to be treated are limited and controversial. If left untreated, clinical symptoms may appear, warranting a second operation, which was consistently associated with technical difficulties, poor outcomes, and psychological and economical burdens[6, 7]. A better therapeutic approach should be taken from the first surgery. Some surgeons may carry out a fusion of the causative segment and simultaneous extensive laminal or semi-laminal decompression surgery or augmenting the vertebral canal in a hidden-proceed manner without fusion at the adjacent segment with asymptomatic preexisting degeneration, but this approach may not be so effective and may even induce symptomatic ASD [4]. Some suggest a complete PLIF at the adjacent level, which may increase not only the costs but also the surgical trauma[8]. Recently, some other surgeons prefer dynamic stabilization, but multiple studies showed that there is no significant advantages of dynamic stabilization on preventing ASD and 
many issues have been reported for the use of dynamic stabilization such as limited indications, dissatisfactory long-term results, and high incidence of reoperation[9-11].

In this paper, we reported a new method to treat severe asymptomatic pre-existing degeneration of adjacent segment by performing a distraction of the intervertebral space and facet fusions in the adjacent segment with significant degeneration. To evaluate the effectiveness of this approach, a retrospective case-control study was conducted.

\section{Methods}

\section{Patient Characteristics}

This study was approved by the Ethics Committee of Honghui Hospital (No. XAHHLLSP0901), and written consent was obtained from all of the patients. We retrospectively reviewed the records of all patients who underwent lumbar spinal fusion surgery in L4/5 and L5/S1 segments for lumbar disc herniation from September 2009 through November 2014. We included the patients who had received imaging examination and preoperative nerve electrophysiological test to confirm that $\mathrm{S} 1$ nerve root was causative of the neurological symptoms, and L5/S1 segment was the causative segment. And if confirmed diagnosis cannot be obtained in the above-mentioned examinations, nerve root block had been conducted.

\section{Inclusion and Exclusion Criteria}

Inclusion criteria were the following: 1) symptomatic degeneration of the L5/S1 segment warranting surgical interference; 2 ) asymptomatic degeneration of the $L 4 / 5$ segment with equal or more than Pfirrmann Grade IV[12] causing equal or more than $25 \%$ spinal canal stenosis (the degree of canal stenosis was evaluated and calculated on preoperative MRI according to the method introduced by Imagama et al[13] as shown in Figure 2b).

Exclusion criteria were the following: 1) symptomatic disc herniation in $L 4 / 5$ segment; 2 ) equal or more than Pfirrmann Grade IV disc degeneration, spondylolisthesis, spinal instability or stenosis in L3/4 segment; 3) history of spinal operation.

The patients who underwent interbody fusion in the causative (L5/S1) segment, and a distraction of the intervertebral space and facet fusions in the adjacent $L 4 / 5$ segment were included as Group $A$, and those who underwent interbody fusions in both L5/S1 and L4/5 segments were included as Group B.

\section{Surgical Technique}

For patients in Group A, standard PLIF was performed in the L5/S1 segment. For the distraction of the intervertebral space in L4/5 segment, disc space between L4 and L5 was moderately distracted using a 
distraction device with the L4 and L5 pedicle screw caps as fulcrums. As a reference for the distraction distance, the height of the adjacent intervertebral space was used, and excessive distraction should be avoided to prevent excessively increased nerve root tension. For the facet fusions in L4/5 segment, Vshape osteotomy was carried out on the L4 inferior articular facet and L5 superior articular facet from top to bottom using an osteotome to create a V-shape groove on the both sides of L4/5 segment (Figure 1a, 1c). The autogenous bone chips from laminectomy were then implanted into the $\mathrm{V}$-shape grooves (Figure 1b, 1d).

For patients in Group B, standard PLIFs were performed in both L5/S1 and L4/5 segments.

Assessment of Outcome

\section{Data Collection and Analysis}

X-rays were obtained at $3,6,9,12,18$, and 24 months and every 1 year thereafter to evaluate the position of the internal fixation, presence of loosening or migration and change in the adjacent L3/4 segment. Symptomatic and functional recovery was evaluated using the Visual Analogue Scale (VAS) and the Oswestry Disability Index (ODI). At the most recent follow-up, X-rays, computed tomography (CT) and MRI were performed.

In Group A, disc height ratio (DHR) of the L4/5 segment was measured according to the Farfan's method[14] on lateral radiograph as shown in Figure 2a. Briefly, DHR was defined as [anterior disc height $(A)+$ posterior disc height (B)] / the distance between the anterior and posterior margins of the disc (C) $[D H R=(A+B) / C]$. The degree of disc degeneration in $L 3 / 4$ and $L 4 / 5$ segments was graded on MRI T2weighted images according to the Pfirrmann grading system[12]. The degree of canal stenosis (DCS) in L3/4 and L4/5 segments was evaluated and calculated on MRI according to the method introduced by Imagama et al[13] as shown in Figure 2b. Briefly, DCS was defined as [the sagittal length of the spinal canal $(A)$ - the maximal sagittal length of the hernia $(B)]$ / the sagittal length of the spinal canal $(A) \times 100$ $[D C S=(A-B) / A \times 100]$. Measurements were performed twice by two observers independently, and interobserver and intraobserver reliabilities of these data were greater than a correlation of 0.80 . Radiographic ASD in L3/4 segment was defined as: 1) development of spondylolisthesis or spinal instability; 2) disc degeneration equal to or more than Pfirrmann Grade IV.

\section{Statistical analysis}

Comparison between preoperative and postoperative values was performed using paired t-test. The independent sample t-test was conducted to compare the outcomes between Groups A and B. Statistical analyses were conducted using SPSS 20.0 (IBM, Armonk, NY, USA). Two-sided $P<0.05$ was considered statistically significant.

\section{Results}




\section{Follow-up of Patient Characteristics}

A total of 103 patients (43 male and 60 female) were included in Group A, whose mean age was 61.5 years (range, 31-74 y). And 81patients (37 male and 44 female) were included in Group B, whose mean age was 59.7 years old (range, 34-72 y). The mean follow-up was 58.5 months (range, $48-75 \mathrm{~m}$ ).

\section{Clinical Results}

As shown in Table 1, compared with Group B, less bleeding ( $315 \pm 84$ vs. $532 \pm 105 \mathrm{ml}$ ), shorter operation time ( $107 \pm 34$ vs. $158 \pm 55 \mathrm{~min})$, and lower costs $(13,830 \pm 2640$ vs. $16,020 \pm 3380$ US\$) (all $P<0.05)$ were found in Group A. The preoperative and final follow-up VAS and ODI scores were presented also in Table 1 , and the clinical symptoms in both groups were improved postoperatively $(P<0.05)$. But preoperatively or postoperatively there was no significant difference of VAS and ODI scores between Groups A and B (all $P>0.05)$. No implant loosening or breakage was found in all patients at the last follow-up.

\section{Radiographic Findings}

In Group A, disc height ratio (DHR) in L4/5 segment was significantly increased from preoperative value of $0.40 \pm 0.13$ to last follow-up value of $0.53 \pm 0.18(P<0.05)$, while the degree of canal stenosis (DCS) was decreased from preoperative value of $34.3 \pm 11.2 \%$ to last follow-up value of $15.9 \pm 9.3 \%(P<0.05)$ (Table 2$)$. Radiographic ASD in L3/4 segment has been found in 13 of 103 (12.62\%) patients in Group A and 9 of $81(11.11 \%)$ patients in Group B at the final follow up, but there was no significant difference in the occurrence of radiographic L3/4 ASD between the two groups ( $P \otimes 0.05)$.

\section{Illustrative Case}

A 54-years-old man with chief complaint of radiating pain to the left lower extremity was diagnosed as lumbar disc herniation. The causative segment had been confirmed to be L5/S1 segment by preoperative examination. Preoperative MRI demonstrated that $42.5 \%$ of spinal canal stenosis in L4/5 segment was caused by the degenerative disc (Pfirrmann Grade IV, Figure 3c, d). We performed a standard PLIF in L5/S1 segment, and distraction of the intervertebral space and facet fusion in L4/5 segment. At the 5year follow-up assessment, CT showed that complete facet fusion in L4-5 facets was achieved (Figure $3 a, 3 b)$, and MRI demonstrated that the degree of spinal canal stenosis in L4/5 segment was decreased to $7.2 \%$ (Figure $3 e, f$ ), indicating that the disc herniation was retracted.

\section{Discussion}

Our study demonstrated that a distraction of the intervertebral space and facet fusions in $L 4 / 5$ segment with severe degeneration, could be effective in preventing ASD of L4/5 segment, and had several 
advantages over interbody fusions in both L5/S1 and L4/5 segments, including decreased bleeding, shorter operation time and lower costs.

The incidence of ASD varies from $8.2 \%$ to $18.5 \%$ among studies, and about $2.7-20 \%$ patients with symptomatic ASD need surgical intervention $[1-3,5]$. Patients with pre-existing degenerative changes at an adjacent motion segment would have a worse long-term clinical outcome and experience a higher rate of sequelae[ $[1,4,6]$. Anandjiwala et al. found that adjacent segments with Pfirrmann Grade IV or V degeneration constitute a significant risk factor for ASD and suggested that adjacent segments with Grade IV or V degeneration should be included in the primary fusion procedure to prevent subsequent ASD [15]. But complete interbody fusion at the adjacent level, not only markedly increases the overall operation costs, but also leads to more surgical trauma with related consequences[8]. Meanwhile, some surgeons choose to augment the vertebral canal in a hidden-proceed manner without fusion at the adjacent segment, but Hikata et al. reported that simultaneous decompression surgery without fusion at the adjacent level may not be effective in reducing the incidence of symptomatic ASD[4].

Interspinous distraction devices and dynamic stabilization across pedicle screws can theoretically prevent ASD by preserving some motion at the segment. Korovessis et al. recommended the use of the Wallis interspinous implant in the unfused vertebral segment cephalad to instrumentation with mild arthritic changes to protect the adjacent segments[16]. In the present study, significant disc degeneration and spinal stenosis in the adjacent segment (L4/5 segment) were present; therefore, distracting the interspinous process is far from enough to retract the herniated disc and decompress the spinal stenosis. Graf ligament and Dynesys device are the mostly used devices for dynamic stabilization. Since there was severe disc degeneration at L4/5 segment, the Graf ligament was unsuitable for our patients[17]. The Dynesys system can resist both tensile and compressive forces and control motion of the segment in all the directions, but this system has a high incidence of screw loosening[18] and the incidence of ASD is similar to that of traditional method[10, 19].

The present study has several limitations. It was a retrospective study that limited the data to those available in the medical charts. Only a single center was involved, and a small sample size of cases were included. Patients with significant L3/4 degeneration were excluded to lower interindividual and intergroup variability, but it could result in lower generalizability. Additional prospective studies are necessary to confirm these results.

\section{Conclusions}

Our outcomes indicate that distraction of the intervertebral space and facet fusions could be effective in treating severe asymptomatic pre-existing degeneration of adjacent segment in lumbar spine.

\section{Abbreviations}


ASD: adjacent segment degeneration; PLIF: posterior lumbar interbody fusion; VAS: Visual Analogue Scale; ODI: Oswestry Disability Index; MRI: magnetic resonance imaging; CT: computed tomography; DHR: disc height ratio; DCS: degree of canal stenosis.

\section{Declarations}

\section{Acknowledgements}

We thank the patients for their cooperation. We would like to acknowledge Dageng Huang for their assistance in reviewing and refining the study protocol.

\section{Funding}

This study was supported by the Postdoctoral Science Foundation in Shaanxi Province of China (NO.2017BSHQYXMZZ15). The funding agency had no input in to the design of the study, the collection, analysis and interpretation of data, the writing of the manuscript, or the decision to publish, and the authors have no competing interests.

\section{Availability of data and materials}

The datasets used and/or analyzed during the current study are available from the corresponding author on reasonable request.

\section{Authors' contributions}

All authors contributed to the analysis and interpretation of data. WJG and DJH has been involved in conception and design. XLZ and JWZ has been involved in drafting the manuscript. ZC, YBL and XDW contributed to the acquisition of data. All authors gave final approval of the version to be published.

\section{Ethics approval and consent to participate}

This study was approved by the Ethics Committee of Honghui Hospital (No. XAHHLLSP0901), and written consent was obtained from all patients.

\section{Consent for publication}

Not applicable

\section{Competing interests}


The authors declare that they have no competing interests.

\section{References}

1.Lee CS, Hwang CJ, Lee SW, Ahn YJ, Kim YT, Lee DH, Lee MY: Risk factors for adjacent segment disease after lumbar fusion. EUR SPINE J 2009, 18(11):1637-1643.

2.Lee JC, Kim Y, Soh JW, Shin BJ: Risk factors of adjacent segment disease requiring surgery after lumbar spinal fusion: comparison of posterior lumbar interbody fusion and posterolateral fusion. Spine (Phila Pa 1976) 2014, 39(5):E339-E345.

3.Imagama S, Kawakami N, Matsubara Y, Tsuji T, Ohara T, Katayama Y, Ishiguro N, Kanemura T: Radiographic Adjacent Segment Degeneration at 5 Years After L4/5 Posterior Lumbar Interbody Fusion With Pedicle Screw Instrumentation: Evaluation by Computed Tomography and Annual Screening With Magnetic Resonance Imaging. CLIN SPINE SURG 2016, 29(9):E442-E451.

4. Hikata T, Kamata M, Furukawa M: Risk factors for adjacent segment disease after posterior lumbar interbody fusion and efficacy of simultaneous decompression surgery for symptomatic adjacent segment disease. J SPINAL DISORD TECH 2014, 27(2):70-75.

5.Park P, Garton HJ, Gala VC, Hoff JT, McGillicuddy JE: Adjacent segment disease after lumbar or lumbosacral fusion: review of the literature. Spine (Phila Pa 1976) 2004, 29(17):1938-1944.

6.Park JY, Chin DK, Cho YE: Accelerated L5-S1 Segment Degeneration after Spinal Fusion on and above L4-5: Minimum 4-Year Follow-Up Results. J Korean Neurosurg Soc 2009, 45(2):81-84.

7.Ghiselli G, Wang JC, Bhatia NN, Hsu WK, Dawson EG: Adjacent segment degeneration in the lumbar spine. J BONE JOINT SURG AM 2004, 86-A(7):1497-1503.

8.Rollinghoff M, Schluter-Brust K, Groos D, Sobottke R, Michael JW, Eysel P, Delank KS: Mid-range outcomes in 64 consecutive cases of multilevel fusion for degenerative diseases of the lumbar spine. Orthop Rev (Pavia) 2010, 2(1):e3.

9.Floman Y, Millgram MA, Smorgick Y, Rand N, Ashkenazi E: Failure of the Wallis interspinous implant to lower the incidence of recurrent lumbar disc herniations in patients undergoing primary disc excision. $J$ SPINAL DISORD TECH 2007, 20(5):337-341.

10.Liu CL, Zhong ZC, Shih SL, Hung C, Lee YE, Chen CS: Influence of Dynesys system screw profile on adjacent segment and screw. J SPINAL DISORD TECH 2010, 23(6):410-417.

11.Verhoof OJ, Bron JL, Wapstra FH, van Royen BJ: High failure rate of the interspinous distraction device (X-Stop) for the treatment of lumbar spinal stenosis caused by degenerative spondylolisthesis. EUR SPINE J 2008, 17(2):188-192. 
12.Pfirrmann CW, Metzdorf A, Zanetti M, Hodler J, Boos N: Magnetic resonance classification of lumbar intervertebral disc degeneration. Spine (Phila Pa 1976) 2001, 26(17):1873-1878.

13.Imagama S, Kawakami N, Matsubara Y, Kanemura T, Tsuji T, Ohara T: Preventive effect of artificial ligamentous stabilization on the upper adjacent segment impairment following posterior lumbar interbody fusion. Spine (Phila Pa 1976) 2009, 34(25):2775-2781.

14.Malmivaara A: Disc degeneration in the thoracolumbar junctional region. Evaluation by radiography and discography in autopsy. ACTA RADIOL 1987, 28(6):755-760.

15.Anandjiwala J, Seo JY, Ha KY, Oh IS, Shin DC: Adjacent segment degeneration after instrumented posterolateral lumbar fusion: a prospective cohort study with a minimum five-year follow-up. EUR SPINE J 2011, 20(11):1951-1960.

16.Korovessis P, Repantis T, Zacharatos S, Zafiropoulos A: Does Wallis implant reduce adjacent segment degeneration above lumbosacral instrumented fusion? EUR SPINE J 2009, 18(6):830-840.

17.Kanayama M, Hashimoto T, Shigenobu K: Rationale, biomechanics, and surgical indications for Graf ligamentoplasty. Orthop Clin North Am 2005, 36(3):373-377.

18.Pham MH, Mehta VA, Patel NN, Jakoi AM, Hsieh PC, Liu JC, Wang JC, Acosta FL: Complications associated with the Dynesys dynamic stabilization system: a comprehensive review of the literature. NEUROSURG FOCUS 2016, 40(1):E2.

19.Kumar A, Beastall J, Hughes J, Karadimas EJ, Nicol M, Smith F, Wardlaw D: Disc changes in the bridged and adjacent segments after Dynesys dynamic stabilization system after two years. Spine (Phila Pa 1976) 2008, 33(26):2909-2914.

\section{Tables}

Table 1. Clinical outcomes in Groups A and B

\begin{tabular}{lll}
\hline & Group A & Group B \\
\hline Bleeding (ml) & $315 \pm 84$ & $532 \pm 105$ \\
Operation time (min) ${ }^{\#}$ & $107 \pm 34$ & $158 \pm 55$ \\
Total cost (US\$) & $13,830 \pm 2640$ & $16,020 \pm 3380$ \\
VAS $^{*}$ & & \\
Preoperative $^{*}$ & $7.7 \pm 2.1$ & $7.5 \pm 1.6$ \\
Final Follow-Up & $1.4 \pm 0.7$ & $1.6 \pm 0.8$ \\
ODI & & \\
Preoperative & $65.3 \pm 11.6$ & $62.2 \pm 8.2$ \\
Final Follow-Up & $13.5 \pm 5.7$ & $14.7 \pm 7.3$ \\
\hline
\end{tabular}


${ }^{\#} P<0.05$ between Groups A and B

${ }^{*} P<0.05$ between preoperative score and final follow-up score in both Groups A and B

VAS: Visual Analog Scale; ODI: Oswestry Disability Index

Table 2. Radiologic Outcomes of L4/5 Segment in Group A

\begin{tabular}{|c|c|c|}
\hline & Preoperative & Final Follow-Up \\
\hline DHR $\#$ & $0.40 \pm 0.13$ & $0.53 \pm 0.18$ \\
\hline DCS \# & $34.3 \pm 11.2 \%$ & $15.9 \pm 9.3 \%$ \\
\hline
\end{tabular}

${ }^{\#} P<0.05$ between preoperative score and final follow-up score

DHR: Disc Height Ratio

DCS: Degree of Canal Stenosis

\section{Figures}

\section{Figure 1}

Figure 1. V-shape osteotomy was carried out on the L4 inferior articular facet and L5 superior articular facet from top to bottom using an osteotome to create a V-shape groove on the both sides of L4/5 segment $(\mathrm{a}, \mathrm{c})$. The autogenous bone chips from laminectomy were then implanted into the $\mathrm{V}$-shape grooves $(b, d)$. 

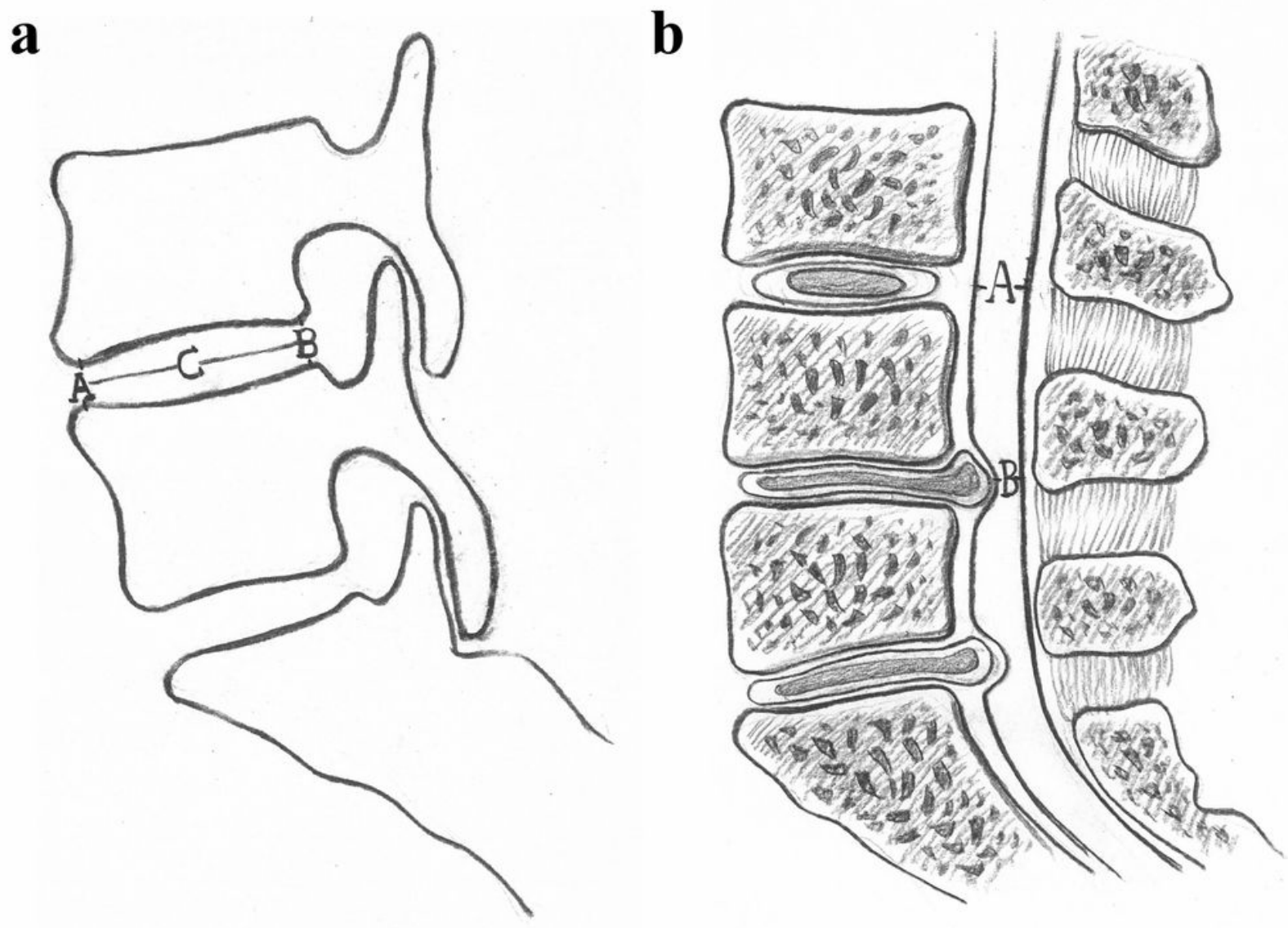

Figure 2

Schematic drawings of the calculations of disc height ratio (DHR) and degree of canal stenosis (DCS). a, $\mathrm{DHR}=(\mathrm{A}+\mathrm{B}) / \mathrm{C} ; \mathrm{b}, \mathrm{DCS}=(\mathrm{A}-\mathrm{B}) / \mathrm{A} \times 100$. 

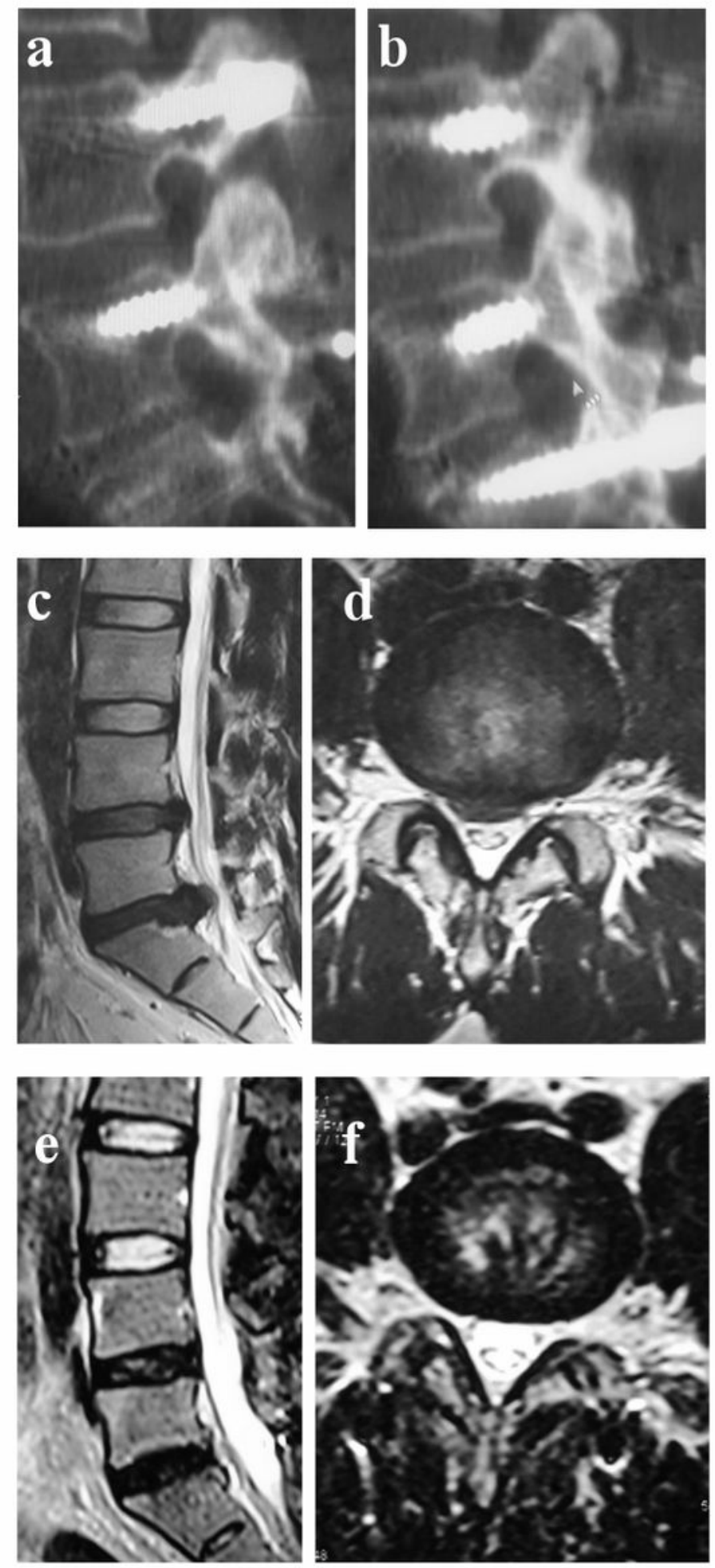

\section{Figure 3}

A 54-year-old patient included in Group A was followed up for 5 years. Preoperative MRI demonstrated severe disc degeneration in L4/5 segment (c, d). A standard PLIF in L5/S1 segment (the causative segment), and a distraction of the intervertebral space and facet fusions in L4/5 segment was performed. At the final follow-up, CT showed that complete fusion in L4-5 facets was achieved (facet of the left side, 
a; right side, b), and MRI demonstrated that the degree of canal stenosis in L4/5 segment was decreased $(e, f)$. 